\title{
Human cytomegalovirus inhibits apoptosis by regulating the activating transcription factor 5 signaling pathway in human malignant glioma cells
}

\author{
TONGMEI WANG, DONGMENG QIAN, MING HU, LING LI, \\ LI ZHANG, HAO CHEN, RUI YANG and BIN WANG \\ Department of Microbiology, Qingdao University Medical College, Qingdao, Shandong 266071, P.R. China
}

Received December 12, 2013; Accepted May 13, 2014

DOI: $10.3892 / \mathrm{ol} .2014 .2264$

\begin{abstract}
The activating transcription factor 5 (ATF5), also termed ATFx, is a member of the ATF/cAMP response element-binding protein (CREB) family of basic zipper proteins. ATF5 is an anti-apoptotic protein that is highly expressed in malignant glioma and is essential for glioma cell survival. Accumulating evidence indicates that human malignant gliomas are universally infected with human cytomegalovirus (HCMV). Recent studies have shown that HCMV may be resistant to the induction of apoptosis by disrupting cellular pathways in glioblastoma. To investigate the potential anti-apoptotic function of HCMV in glioma, malignant U87 glioma cells were infected with HCMV. The present study showed that HCMV infection suppressed apoptosis in glioblastoma U87 cells by regulating the expression of ATF5. Furthermore, in glioblastoma U87 cells, HCMV infection induced cellular proliferation in parallel with an increase in the expression level of ATF5 and B-cell lymphoma/leukemia-2 to $\mathrm{Bcl}-2$-associated $\mathrm{X}$ protein ratio. Loss of ATF5 function was achieved using a dominant-negative form of ATF5 in U87 cells, whereby cells appeared to grow marginally following HCMV infection when compared with the control. However, the anti-apoptotic ability was appeared to decline in the terminal deoxynucleotidyl transferase-mediated
\end{abstract}

Correspondence to: Professor Bin Wang, Department of Microbiology, Qingdao University Medical College, 308 Ningxia Road, Qingdao, Shandong 266071, P.R. China E-mail: qdmblm@163.com

Abbreviations: ATF5, activating transcription factor 5; Bcl-2, B-cell lymphoma/leukmia-2; BAX, Bcl-2-associated $\mathrm{X}$ protein; $\mathrm{CREB}$, cAMP response element-binding protein; HCMV, human cytomegalovirus; IE, immediate early; MTT, 3-(4,5-dimethylthiazol-2-yl)-2,5-diphenyltetrazolium bromide; PBS, phosphate-buffered saline; TUNEL, terminal deoxynucleotidyl transferase-mediated dUTP nick end labeling

Key words: glioblastoma cells, activating transcription factor 5, apoptosis, human cytomegalovirus infection
dUTP nick end labeling assay. These results indicate that ATF5 signaling pathways may be important in the anti-apoptotic activity of HCMV-infected glioblastoma cells; therefore, the anti-apoptotic molecular mechanisms of HCMV in human glioblastoma cells were investigated in the current study. Prevention of HCMV infection may present a potential and promising approach for the treatment of malignant gliomas.

\section{Introduction}

Glioblastomas are a particularly devastating form of primary brain tumor. Due to the highly infiltrative and invasive nature of such tumors, it remains clinically intractable. Patient survival time is generally only 12-18 months even following surgical resection with subsequent radiation and chemotherapy (1-3). Recent studies have indicated that the development of a tumor is often associated with the regulation of a variety of transcription factors $(1,4,5)$. Activating transcription factor 5 (ATF5) is a novel factor that is closely associated with tumor cell differentiation, proliferation and apoptosis. ATF5 is a member of the ATF/cAMP responsive element-binding (CREB) family of transcription factors, which includes a large group of basic leucine zipper proteins that mediate diverse transcriptional regulatory functions (6-9). ATF5, an anti-apoptotic factor, is highly expressed in malignant glioma and is important in the promotion of cell survival (5). ATF5 loss of function induces apoptosis in a number of glioma and breast cancer cell lines $(10,11)$, however, interfering with ATF5 function in non-tumor brain cells has not been found to affect their survival (10). Dluzen et al (12) demonstrated that B-cell lymphoma/leukemia-2 (Bcl-2) is a downstream target of ATF5 in gliomas and breast cancer. The Bcl-2 family of proteins includes anti-apoptotic proteins, such as Bcl-2, Bcl-Xl and induced myeloid leukemia cell differentiation protein, and apoptotic proteins, such as Bcl-2 homologous antagonist/killer, Bcl-2 associated $\mathrm{X}$ protein (BAX), BH3 interacting-domain and B-cell lymphoma 2 interacting mediator of cell death. The regulation and balance of the Bcl-2 family proteins in a particular cell results in the inhibition or induction of apoptotic signaling pathways (12-14).

The human cytomegalovirus (HCMV) infection has been detected in malignant gliomas in a high percentage of cases, 
although not in the adjacent healthy brain tissues (15). Growing evidence indicates that HCMV infection may increase the malignancy of infected cells by disrupting cellular pathways, such as apoptosis (16-18). Apoptosis is detrimental to HCMV, as it functions as a cellular antivirus response to eliminate infected cells (by activating the immune response) or is deleterious (an inevitable consequence of the stress that is inflicted by viruses on host cells). To survive, these viruses have developed numerous strategies to prevent the premature cell death of host cells (19,20). HCMV infection in glial cells that does not lead to cell apoptosis may promote clonal expansion without producing a productive or cytopathic virus infection. Long-term persistence of HCMV in malignant glioma cells may result in the occurrence of variant strains, which exhibit a minimal cytopathic effect, and therefore, HCMV may be reactivated in latently infected glioma cells when cells are exposed to inflammatory stimuli or superinfected with other HCMV strains $(21,22)$. The sustained expression of specific HCMV gene products may promote the overall glioma phenotype, as HCMV encodes for gene products that regulate cellular pathways involved in mutagenesis and apoptosis, and host antitumor immune responses (23). HCMV immediate-early (IE) genes 1 and 2 are the first set of viral genes that are activated within HCMV-infected cells (24). IE1 and IE2 proteins regulate transcription of viral and cellular genes within HCMV-infected cells (25). In addition, the IE protein has a binding site for the ATF/CREB family of transcription factors, which upon binding forms a complex to activate downstream elements (26).

Due to the high prevalence of HCMV and ATF5 expression observed in vivo in human malignant glioma, the aim of the present study was to investigate the role of the ATF5 signaling pathway in HCMV-infected glioblastoma cells.

\section{Materials and methods}

Cell lines and viruses. Human glioblastoma U87 cell lines were purchased from the Shanghai Cell Resource Center of the Chinese Academy of Sciences (Shanghai, China). U87 cells were propagated in HyClone ${ }^{\mathrm{TM}}$ Minimum Essential Medium with $10 \%$ fetal bovine serum (Thermo Fisher Scientific Inc., Rockford, IL, USA) and maintained at $37^{\circ} \mathrm{C}$ in a humidified atmosphere containing $5 \%(\mathrm{v} / \mathrm{v}) \mathrm{CO}_{2}$. $\mathrm{HCMV}$ AD169 (France Pasteur Laboratory, Paris, France) was tittered by plaque titration in human embryonic lung fibroblast cells and expressed as the number of plaque-forming units per milliliter. The HCMV was propagated in human embryonic lung fibroblast cells with serum-free medium and the cell supernatant was harvested and stored at $-80^{\circ} \mathrm{C}$.

Loss of ATF5 expression or function achieved using small interfering (si) RNA or a dominant-negative (dn) form of ATF5. To establish U87 cell lines with a stable knockdown of ATF5, a GV113 control plasmid and three GV113 plasmids containing ATF5 lentivirus short hairpin RNAs, LV-ATF5-RNAi (8842-1; GCGAGATCCAGTACGTCAA), LV-ATF5-RNAi(8843-1;TCTTGGATACTCTGGACTT) and LV-ATF5-RNAi (8844-1;TGGAACAGATGGAAGACTT) (Shanghai GeneChem Co., Ltd., Shanghai, China), targeting the ATF5 coding sequence were separately transduced into U87 cells.
The pLeGFP-C1-NTAzip-ATF5 plasmid was transfected to block the function of ATF5 simultaneously. Cells were transfected with pLeGFP-C1-NTAzip-ATF5 and the pLeGFP-C1 plasmid using Lipofectamine 2000 (Invitrogen Life Technologies, Carlsbad, CA, USA). To analyze proliferation and apoptosis and to perform western blot assays, cells were collected at $0,12,24$ and $48 \mathrm{~h}$ following transfection.

3-(4,5-Dimethylthiazol-2-yl)-2, 5-diphenyltetrazolium bromide (MTT) assay. Cell proliferation was determined using the MMT assay (Sigma-Aldrich, St. Louis, MO, USA). Glioblastoma U87 cells were plated in 96-well microplate formats according to the manufacturer's instructions. Cell lines were seeded in five replication wells at 5,000 cells/well and cultured for $0,12,24$ or $48 \mathrm{~h}$. Following MTT uptake for a duration of $4 \mathrm{~h}$, cells were lysed in $150 \mu \mathrm{l}$ dimethyl sulfoxide and absorbance was measured at $475 \mathrm{~nm}$ using a fluorescence microplate reader (Sunrise Remote, Tecan Austria GmbH, Grödig, Austria).

RNA extraction, and quantitative polymerase chain reaction $(q P C R)$. RNA was extracted using TRIzol reagent [Takara Biotechnology (Dalian) Co., Ltd., Dalian, China] according to the manufacturer's instructions. To produce cDNAs, $1 \mu \mathrm{g}$ RNA was reverse-transcribed using the PrimeScript RT reagent kit with gDNA eraser (Perfect Real Time; Takara Biotechnology [Dalian] Co., Ltd.) according to the manufacturer's instructions. qPCR analyses were performed using the GoTaq qPCR Master Mix (Promega Corporation, Madison, WI, USA). The following primers were used: Forward, 5'-AGTGGGCTGGGATGGCTCGTAGAC-3' and reverse, 5'-CTCGGGTGGTGGCAGGATGTGG-3' for ATF5; forward, 5'-GCGCAATATCATGAAAGATAAGAACA-3' and reverse, 5'-GATTGGTGTTGCGGAACATG-3' for IE2; forward, 5'-CTGCACCTGACGCCCTTCACC-3' and reverse, 5'-CACATGACCCCACCGAACTCAAAGA-3' for Bcl-2; forward, 5'-TGGAGCTGCAGAGGATGATTG-3' and reverse, 5'-GAAGTTGCCGTCAGAAAACATG-3' for BAX; and forward, 5'-TGGAACGGTGAAGGTGACAG-3' and reverse, 5'-GGCTTTTAGGATGGCAAGGG-3' for $\beta$-actin.

Western blot analysis. Cells were washed three times with ice-cold phosphate-buffered saline (PBS). Next, cells were dissolved in $500 \mu 1$ lysis buffer and $5 \mu 1$ phenylmethylsulfonyl fluoride (Beyotime Institute of Biotechnology, Shanghai, China). Samples were centrifuged (Eppendorf 5804R, Eppendorf Corporation, Hamburg, Germany) at 20,100 x g for $5 \mathrm{~min}$ at $4^{\circ} \mathrm{C}$ to separate the membrane fraction from the cytosolic fraction. After boiling for $5 \mathrm{~min}$, equivalent amounts of protein $(30 \mu \mathrm{g})$ were resolved by $12 \%$ SDS-PAGE, electroblotted onto polyvinylidene fluoride membrane (Millipore, Billerica, MA, USA) and immunoreacted overnight with IE genes (Virostat, Inc., Westbrook, ME, USA), ATF5 (Abcam, Cambridge, UK), Bcl-2 and Bax (Bioss, Inc., Woburn, MA, USA), followed by a 2-h incubation with the horseradish peroxidase-conjugated secondary antibody (Bioss, Inc.). Chemiluminescent signals were generated by the SuperSignal West Pico Trial Kit (Thermo Fisher Scientific Inc.) and detected using the Vilber Lourmat imaging system (Vilber Lourmat Corporation, Torcy, France). 
Terminal deoxynucleotidyl transferase-mediated dUTP nick end labeling (TUNEL) assay. To detect the apoptosis rate of HCMV-infected glioblastoma cells, an in situ cell death detection kit (Roche Diagnostics Corporation, Indianapolis, IN, USA) was used. Cells grown in 6-well culture clusters were treated with HCMV for $48 \mathrm{~h}$. Treated cells were fixed onto poly-(L-lysine) coated slides with $4 \%$ paraformaldehyde. The slides were rinsed with PBS and the cells were permeabilized with 0.1\% Triton X-100 [Sangon Biotech (Shanghai) Co., Ltd., Shanghai, China]. Then, the slides were washed with PBS and the cells were incubated in $50 \mu \mathrm{l}$ TUNEL reaction mixture for $60 \mathrm{~min}$ at $37^{\circ} \mathrm{C}$ in the dark. Next, $50 \mu \mathrm{l}$ converter-POD (Roche, Basel, Switzerland) was added and incubated for $30 \mathrm{~min}$ at $37^{\circ} \mathrm{C}$ in a humidified chamber. Slides were rinsed with PBS. Next, $50 \mu \mathrm{l}$ 3,3'-diaminobenzidine substrate was added and incubated for $10 \mathrm{~min}$ at $20^{\circ} \mathrm{C}$. A total of $50 \mu 1$ hematoxylin was then added and incubated for $3 \mathrm{~min}$ at room temperature. The slides were mounted under a glass coverslip with PBS and analyzed under a light microscope (Motic B1-223A, Motic Deutschland GmbH, Wetzlar, Germany).

Statistical analysis. Data are presented as the mean \pm standard deviation. Statistical analysis was performed using the Student's t-test and $\mathrm{P}<0.05$ was considered to indicate a statistically significant difference.

\section{Results}

Effect of HCMV infection on cell proliferation and expression of ATF5, Bcl-2 and BAX. To determine the role of ATF5 expression in the HCMV-infected U87 glioblastoma cells, the proliferation ratio of U87 cells was examined in response to HCMV infection. Compared with normal U87 cells, proliferation was enhanced by HCMV infection (Fig. 1A). This observation indicated that HCMV infection promotes growth proliferation in U87 glioblastoma cells. To elucidate the underlying mechanisms, the expression of ATF5 in HCMV-infected U87 cells was examined. As shown in Fig. 1B, ATF5 mRNA was upregulated $12 \mathrm{~h}$ following HCMV infection and continued to increase until $48 \mathrm{~h}$ following infection. Similarly, ATF5 protein levels were upregulated (Fig. $1 \mathrm{C}$ and $\mathrm{D} ; \mathrm{P}<0.05$ ). These results demonstrated that HCMV infection upregulates ATF5 expression in U87 cells. Studies have shown that anti-apoptotic Bcl-2 is regulated by ATF5 in glioblastoma cells and BAX is an apoptosis member of the $\mathrm{Bcl}-2$ family. To examine the expression of Bcl-2 and BAX in HCMV-infected U87 cells, qPCR and western blot analysis were performed. As shown in Fig. 1C-E, the ratio of $\mathrm{Bcl}-2$ to $\mathrm{BAX}$ increased in U87 cells treated with $\mathrm{HCMV}(\mathrm{P}<0.05)$. In addition, $\mathrm{Bcl}-2$ protein levels were upregulated in U87 cells treated with HCMV (Fig. 1C and D; $\mathrm{P}<0.05$ ). These results indicated that HCMV infection enhances the anti-apoptotic ability of U87 cells.

Interfering with ATF5 affects HCMV-infected U87 cell proliferation. To investigate the function of ATF5 in HCMV-infected U87 cells, the effect of silencing ATF5 on the proliferation of HMCV-infected U87 cells was examined. U87 cells were infected with lentiviral RNAi to interfere with ATF5. Western blot analysis revealed that ATF5 protein levels were markedly decreased (data not shown). The cells were subsequently infected with HCMV at various time points and cell proliferation was determined by MTT assay. As shown in Fig. 2A, compared with U87 cells, cell proliferation was reduced following ATF5 interference in U87 cells $(\mathrm{P}<0.05$; between 12 and $48 \mathrm{~h})$. Cell viability following ATF5 interference in U87 cells was marginally increased following HCMV infection when compared with siATF5 U87 cells. To further validate this observation, U87 cells were transfected with dnATF5 plasmids to interfere with ATF5. As shown in Fig. 2B, the results were consistent with Fig. 2A. These results indicated that ATF5 may be involved in the regulation of cell proliferation of HCMV-infected U87 cells in vitro.

Analysis of apoptosis in dnATF5 U87 treated with HCMV using TUNEL. Previous studies have demonstrated that HCMV may increase the malignancy of glioma by blocking apoptosis. ATF5 is closely associated with tumor cell apoptosis. In addition, the present study found that HCMV infection regulates the expression of ATF5 in glioma. It is hypothesized that anti-apoptosis of HCMV infection may be associated with ATF5 pathways. In siATF5 U87 cells, almost no apoptotic cells were identified. However, apoptosis occurred in U87 cells, which were transfected with dnATF5 plasmids. Furthermore, apoptotic cell death was detected in $40 \%$ of dnATF5 U87 cells. The number of TUNEL-positive cells (dead cells) decreased by $4-5 \%$ following HCMV infection. As shown in Fig. 3, TUNEL-positive cells were not observed in normal or U87 cells (Fig. 3A and B), however, TUNEL-positive cells were detected in dnATF5 U87 cells (Fig. 3C). Following HCMV infection for $48 \mathrm{~h}, 40 \%$ of TUNEL-positive cells were detected in dnATF5 U87 cells whereas 35\% of TUNEL-positive cells were detected in HCMV-infected dnATF5 cells (Fig. 3G). Compared with the control, in U87 cells, which had lost ATF5 function, no significant difference was identified in the decline of TUNEL-positive cells (Fig. 3G). These results support the hypothesis that the anti-apoptotic effect of HCMV infection is associated with ATF5 expression.

$B c l-2$ and BAX expression following interference with ATF5 in HMCV-treated U87 cells. Bcl-2 is a downstream target of ATF5. Interaction of BAX and Bcl-2 is important in the regulation of apoptosis. In the present study, it was found that HCMV infection in U87 cells regulated the expression of ATF5. Furthermore, following interference with ATF5 in U87 cells, the anti-apoptotic ability was decreased following HCMV infection. The expression of anti-apoptotic Bcl-2 and apoptotic BAX protein was further detected in HCMV-infected dnATF5 U87 cells by western blot analysis. In dnATF5 U87 cells, changes in $\mathrm{Bcl}-2$ and $\mathrm{BAX}$ protein expression following HCMV infection are shown in Fig. 4. Compared with the control group, in HCMV-infected U87 cells, which had lost ATF5 expression, no significant differences in Bcl-2 and BAX protein levels between 0 and $48 \mathrm{~h}$ were identified.

\section{Discussion}

Increasing evidence implicates infectious agents as causal factors in the development of human cancers. Infectious agents may be promoters of neoplastic transformation. For example, HCMV may modify host cell transcription to prolong 
A

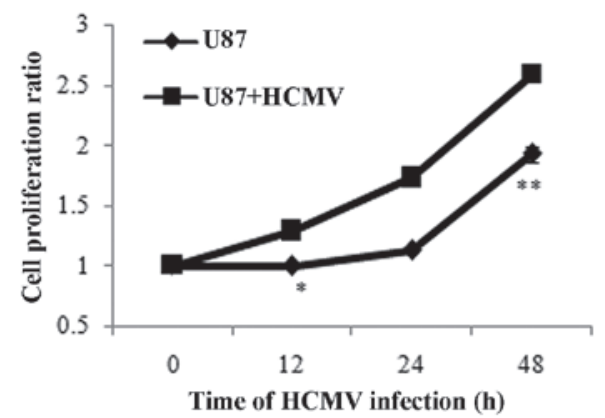

C



B

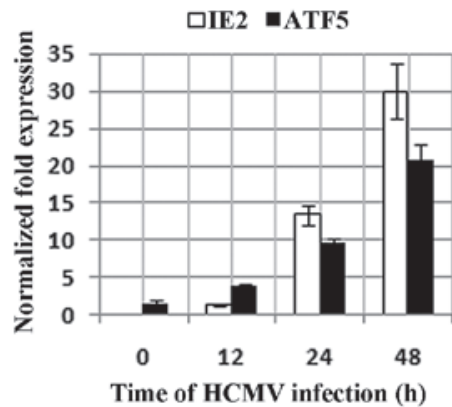

D

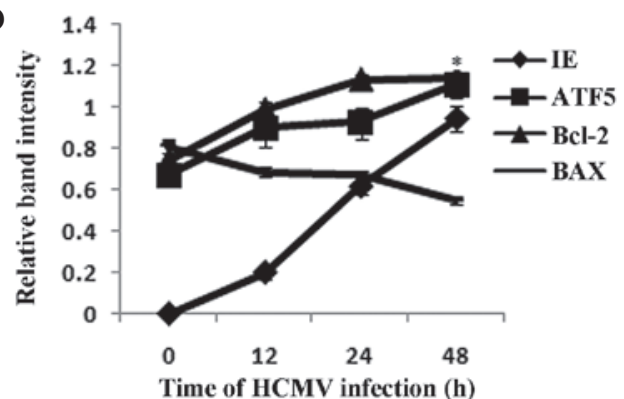

$\mathbf{E}$

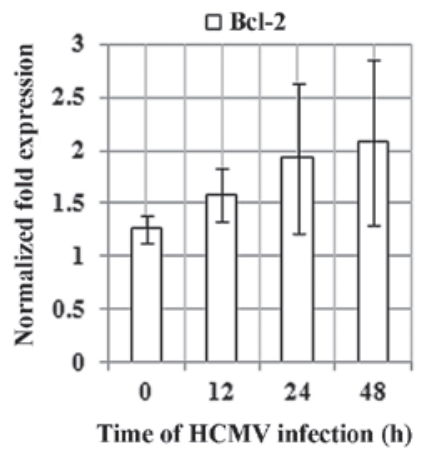

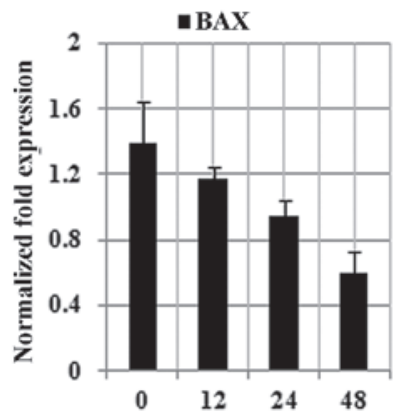

Time of HCMV infection (h)

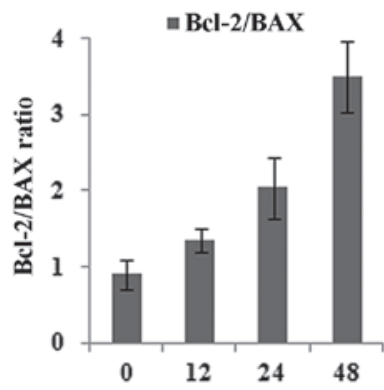

Time of HCMV infection (h)

Figure 1. Effect of HCMV infection on cell proliferation and expression of ATF5, Bcl-2 and BAX in U87 cells. (A) Cell proliferation was measured using a 3-(4,5-dimethylthiazol-2-yl)-2,5-diphenyltetrazolium bromide assay. Data are presented as the mean \pm SD of three independent experiments ("P $<0.05$ and ${ }^{* *} \mathrm{P}<0.01$ vs. U87 + HCMV). (B) qPCR analyses showing the expression levels of IE2 and ATF5 mRNA in U87 cells following HCMV infection for 0, 12,24 and $48 \mathrm{~h}$. (C) Western blot analysis of IE genes, ATF5, Bcl-2 and BAX protein in HCMV-infected U87 cells. (D) Relative expression of IE, ATF5, Bcl-2 and BAX, all vs. $\beta$-actin in U87 cells infected with HCMV for $0,12,24$ and $48 \mathrm{~h}$, according to the results of figure $1 \mathrm{C}$. Data are presented as the mean $\pm \mathrm{SD}$ ("P $<0.01$ vs. $0 \mathrm{~h}$ ATF5 expression). (E) qPCR analyses of Bcl-2 and BAX mRNA expression in U87 cells following HCMV infection, where the ratio of Bcl-2/BAX was calculated. HMCV, human cytomegalovirus; ATF5, activating transcription factor 5; Bcl-2, B-cell lymphoma/leukmia-2; BAX, Bcl-2-associated X protein; $\mathrm{SD}$, standard deviation; qPCR, quantitative polymerase chain reaction; IE, immediate-early.
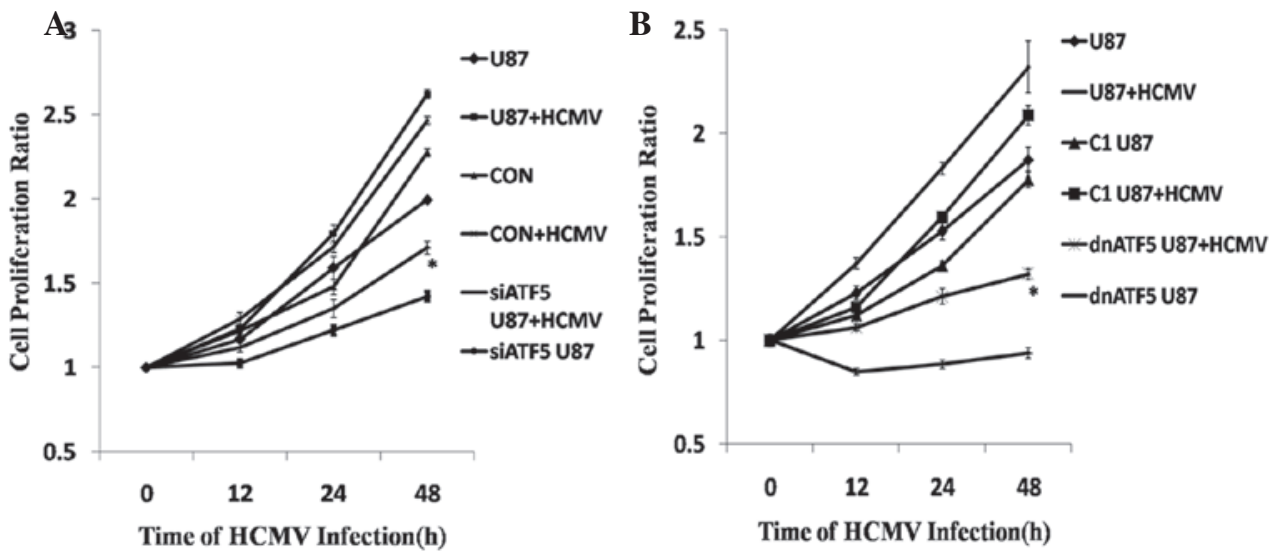

Figure 2. Cell viability analysis of siATF5 or dnATF5 U87 cells following HCMV infection. (A) U87 cells expressing non-targeting siRNA or ATF5 short hairpin RNA were infected with HCMV for 0, 12, 24 or 48 h. Knocking down ATF5 expression reduced tumor cell proliferation. HCMV-infected siATF5 U87 marginally promoted proliferation. (B) U87 cells were produced with loss of ATF5 function using a dn form. The results were consistent with siATF5 U87 cells. Cell proliferation was measured using 3-(4,5-dimethylthiazol-2-yl)-2,5-diphenyltetrazolium bromide assay. Data are presented as the mean \pm standard deviation of three independent experiments ( $\mathrm{P}<0.05$ vs. con + HCMV). si, small interfering; dn, dominant-negative; ATF5, activating transcription factor 5; HCMV, human cytomegalovirus; con, control. 

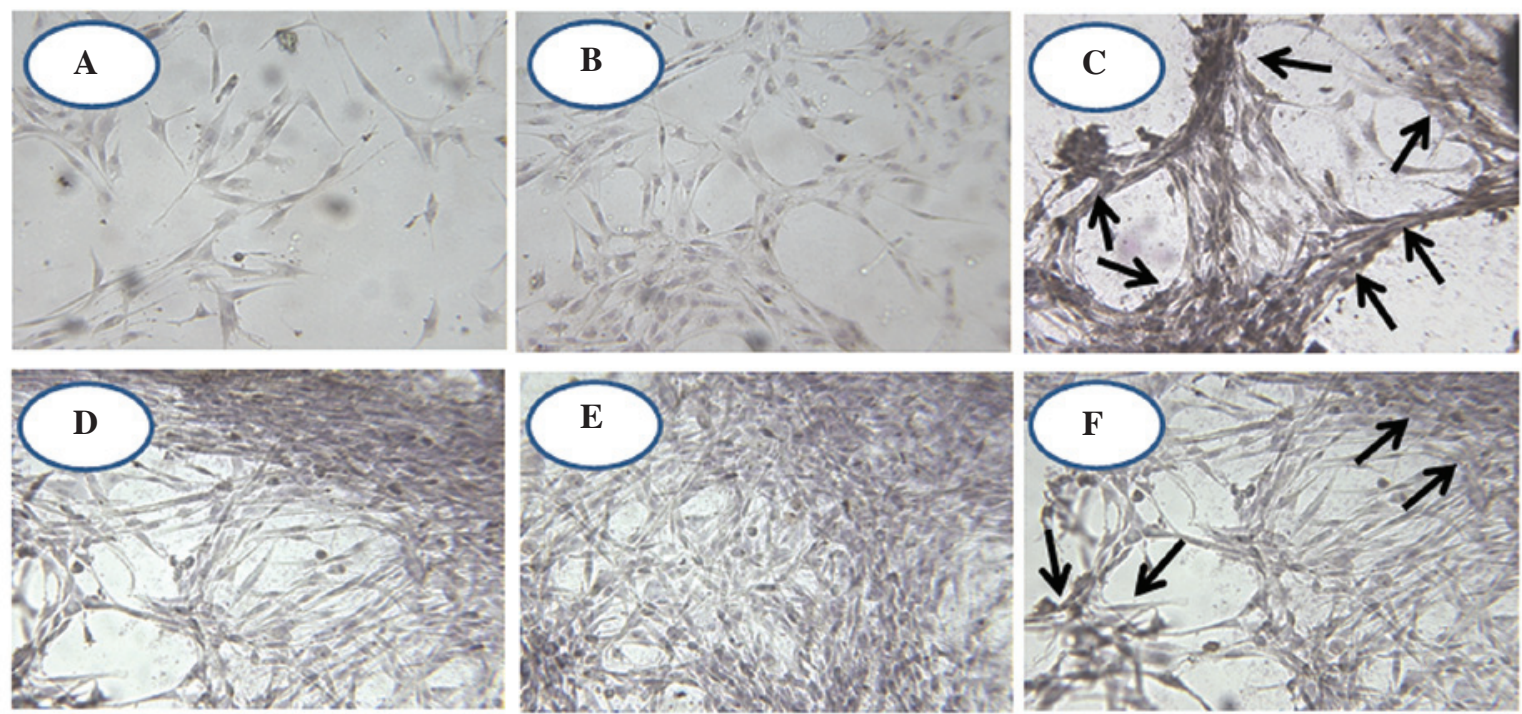

G

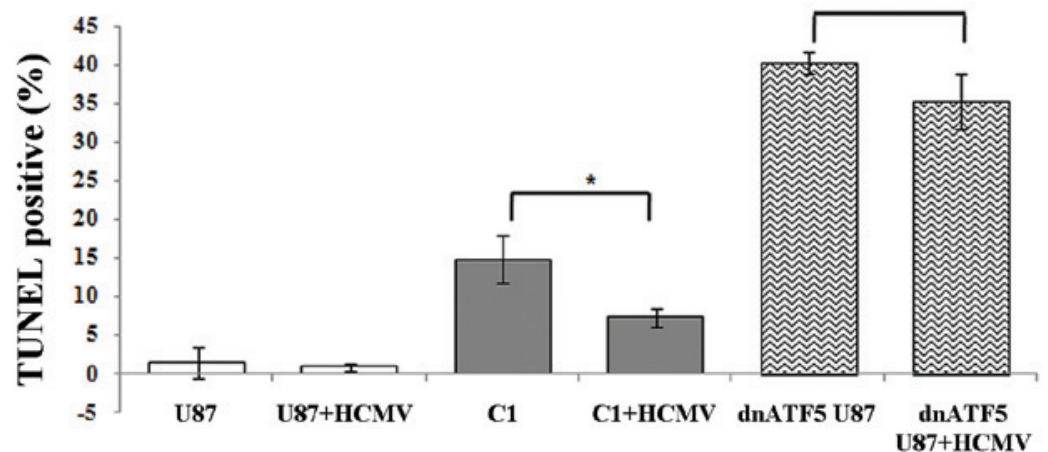

Figure 3. Apoptotic analysis of U87 cells exhibiting a loss of ATF5 function and treatment with or without HCMV by TUNEL assay and hematoxylin staining. (A) U87 cells and (B) HCMV-infected U87 cells did not demonstrate apoptosis. (C) Interfering with ATF5 function resulted in U87 cell apoptosis (as shown by arrows) and this data was consistent with previous studies (31). (D) U87 cells were transfected with pLeGFP-C1 plasmids using Lipofectamine 2000. A significant decrease was observed in (E) HCMV-infected control and TUNEL-positive cells. (C) U87 cells were transfected with the pLeGFP-C1-NTAzip-ATF5 plasmid and apoptosis was evident. (F) HCMV-infected U87 cells with ATF5 loss of function exhibited a decrease in TUNEL-positive cells. Magnification, 400. (G) Bar chart showing the mean apoptosis index per slide (A-F;" P<0.05). ATF5, activating transcription factor 5; HMCV, human cytomegalovirus; dn, dominant-negative; TUNEL, terminal deoxynucleotidyl transferase-mediated dUTP nick end labeling.



Figure 4. Western blot analysis showing changes in Bcl-2 and BAX protein levels in dnATF5 U87 cells treated with HCMV. The expression of the Bcl-2 and BAX protein was not altered following HCMV infection between 0 and $48 \mathrm{~h}$ in dnATF5 U87 cells when compared with the control. dnATF5, dominant-negative activating transcription factor 5; Bcl-2, B-cell lymphoma/leukmia-2; BAX, Bcl-2-associated X protein; HMCV, human cytomegalovirus.

its replication by establishing a latent infection. However, the modification may cause significant morbidity of cell life. Glioblastoma multiforme (GBM) is a malignant and lethal brain cancer of unknown origin, and the majority of cases are resistant to radiotherapy and chemotherapy (27). Over the past decade, various studies have searched for the presence of HCMV in GBM samples (23) and Cobbs et al (15) initially reported the expression of HCMV proteins and oligonucleotides in a high percentage of gliomas. Growing evidence that HCMV is specifically detected in a variety of human malignancies at low levels of expression indicates that the virus may facilitate the neoplastic process in malignancy via oncomodulation. In addition, oncomodulation implies that HCMV infects established tumor cells and increases their malignant potential without necessarily being oncogenic (16). Therefore, it is important to understand how this virus modifies the host.

Viruses have acquired the capacity to modify the host environment to enable the successful completion of their life cycle. The use and control of the cellular transcriptional machinery are among the most important functions of viruses. For 
example, apoptosis of infected cells may limit viral replication and thus serves as an innate defense mechanism against viral infection. Consequently, viruses delay apoptosis by affecting anti-apoptotic proteins. Recent studies have confirmed that HCMV establishes a replication-favorable environment to avoid prematurely compromising the cell's ability to produce viral progeny (28). In addition, our previous studies have also confirmed that HCMV infection inhibits tumor necrosis factor- $\alpha$ induced apoptosis (29).

The present study revealed that HCMV infection blocks apoptosis in glioblastoma U87 cells and increases the expression levels of the ATF5 and Bcl-2 to BAX ratio. ATF5, a member of the ATF/CREB family of basic leucine zipper proteins, is an anti-apoptotic protein, which is highly expressed in malignant glioma, but not in normal brain tissues, and is essential for the survival of glioma cells. Our previous studies demonstrated that ATF5 is highly expressed in epithelial ovarian carcinomas and human pancreatic carcinomas, compared with healthy ovarian and pancreatic tissues (30,31). Although ATF5 is critically involved in cell survival, cell proliferation and differentiation, the upstream mechanism that regulates ATF5 function remains unclear.

The results of the current study revealed that the ATF5 signaling pathway is involved in the anti-apoptotic effects that are induced by HCMV infection in U87 cells. Previous studies have shown that ATF5 is an important anti-apoptotic protein in malignant glioma. However, to the best of our knowledge, no studies have shown that the anti-apoptotic effect of HCMV infection in malignant glioma is associated with ATF5. The results of the present study demonstrated that HCMV infection in U87 cells promotes cell proliferation and upregulates the expression of ATF5. In addition, when interfering with ATF5 in U87 cells, the anti-apoptotic ability was decreased following HCMV infection (Fig. 3). In revealing the anti-apoptotic mechanism of ATF5 in HCMV-infected glioma, it was found that Bcl-2 was downregulated in the U87 cells in which ATF5 was inhibited, when compared with the control (Fig. 4). Furthermore, previous studies have shown that Bcl-2 is a downstream target of ATF5 that mediates the pro-survival function of ATF5 in C6 glioma and MCF-7 breast cancer cells. ATF5 binds to an ATF5-specific regulatory element that is downstream of and adjacent to the negative regulatory element in the Bcl-2 P2 promoter, stimulating Bcl-2 expression (12) and the results of the present study were consistent with this. These results provide a novel insight into the influence of HCMV infection on tumor cell apoptosis.

In conclusion, the present study found that ATF5 is involved in the anti-apoptotic effect induced in HCMV-infected U87 cells. HCMV infection promotes the expression of ATF5 and elevates the Bcl-2 to BAX ratio and enhances anti-apoptosis in U87 cells. Furthermore, ATF5 knockdown may reduce the anti-apoptotic ability of HCMV-infected U87 cells. These results facilitate the understanding regarding the influence of HCMV infection on glioma cell apoptosis and thus, HCMV may present a potential therapeutic target.

\section{Acknowledgements}

The present study was supported by the National Natural Science Foundation of China (grant nos. 81070501 and 30770105), the Natural Science Foundation of Shandong
Province (grant no. J122211) and the American Cancer Society and the U.S. Department of Defense (D.X.L.).

\section{References}

1. Arias A, Lamé MW, Santarelli L, Hen R, Greene LA and Angelastro JM: Regulated ATF5 loss-of-function in adult mice blocks formation and causes regression/eradication of gliomas. Oncogene 31: 739-751, 2012.

2. Stupp R, Mason WP, van den Bent MJ, et al; European Organisation for Research and Treatment of Cancer Brain Tumor and Radiotherapy Groups; National Cancer Institute of Canada Clinical Trials Group: Radiotherapy plus concomitant and adjuvant temozolomide for glioblastoma. N Engl J Med 352: 987-996, 2005.

3. Vescovi AL, Galli R and Reynolds BA: Brain tumour stem cells. Nat Rev Cancer 6: 425-436, 2006.

4. Michaelis M, Doerr HW and Cinatl J: The story of human cytomegalovirus and cancer: increasing evidence and open questions. Neoplasia 11: 1-9, 2009.

5. Sheng Z, Li L, Zhu LJ, et al: A genome-wide RNA interference screen reveals an essential CREB3L2-ATF5-MCL1 survival pathway in malignant glioma with therapeutic implications. Nat Med 16: 671-677, 2010.

6. Persengiev SP and Green MR: The role of ATF/CREB family members in cell growth, survival and apoptosis. Apoptosis 8: 225-228, 2003.

7. Watatani Y, Ichikawa K, Nakanishi N, et al: Stress-induced translation of ATF5 mRNA is regulated by the 5'-untranslated region. J Biol Chem 283: 2543-2553, 2008.

8. Angelastro JM, Ignatova TN, Kukekov VG, et al: Regulated expression of ATF5 is required for the progression of neural progenitor cells to neurons. J Neurosci 23: 4590-4600, 2003.

9. Persengiev SP, Devireddy LR and Green MR: Inhibition of apoptosis by ATFx: a novel role for a member of the ATF/CREB family of mammalian bZIP transcription factors. Genes Dev 16: 1806-1814, 2002.

10. Angelastro JM, Canoll PD, Kuo J, et al: Selective destruction of glioblastoma cells by interference with the activity or expression of ATF5. Oncogene 25: 907-916, 2006.

11. Monaco SE, Angelastro JM, Szabolcs M and Greene LA: The transcription factor ATF5 is widely expressed in carcinomas, and interference with its function selectively kills neoplastic, but not nontransformed, breast cell lines. Int J Cancer 120: 1883-1890, 2007.

12. Dluzen D, Li G, Tacelosky D, Moreau M and Liu DX: BCL-2 is a downstream target of ATF5 that mediates the prosurvival function of ATF5 in a cell type-dependent manner. J Biol Chem 286: 7705-7713, 2011.

13. Chipuk JE, Moldoveanu T, Llambi F, Parsons MJ and Green DR: The BCL-2 family reunion. Mol Cell 37: 299-310, 2010.

14. Harris MH and Thompson CB: The role of the Bcl-2 family in the regulation of outer mitochondrial membrane permeability. Cell Death Differ 7: 1182-1191, 2000.

15. Cobbs CS, Soroceanu L, Denham S, Zhang W and Kraus MH: Modulation of oncogenic phenotype in human glioma cells by cytomegalovirus IE1-mediated mitogenicity. Cancer Res 68: 724-730, 2008.

16. Barami K: Oncomodulatory mechanisms of human cytomegalovirus in gliomas. J Clin Neurosci 17: 819-823, 2010.

17. Michaelis M, Kotchetkov R, Vogel JU, Doerr HW and Cinatl J Jr: Cytomegalovirus infection blocks apoptosis in cancer cells. Cell Mol Life Sci 61: 1307-1316, 2004.

18. Ranganathan P, Clark PA, Kuo JS, Salamat MS and Kalejta RF: Significant association of multiple human cytomegalovirus genomic Loci with glioblastoma multiforme samples. J Virol 86: 854-864, 2012.

19. Xuan B, Qian Z, Torigoi E and Yu D: Human cytomegalovirus protein pUL38 induces ATF4 expression, inhibits persistent JNK phosphorylation, and suppresses endoplasmic reticulum stress-induced cell death. J Virol 83: 3463-3474, 2009.

20. Benedict CA, Norris PS and Ware CF: To kill or be killed: viral evasion of apoptosis. Nat Immunol 3: 1013-1018, 2002.

21. Poland SD, Costello P, Dekaban GA and Rice GP: Cytomegalovirus in the brain: in vitro infection of human brain-derived cells. J Infect Dis 162: 1252-1262, 1990.

22. Ogura T, Tanaka J, Kamiya S, Sato H, Ogura H and Hatano M: Human cytomegalovirus persistent infection in a human central nervous system cell line: production of a variant virus with different growth characteristics. J Gen Virol 67: 2605-2616, 1986. 
23. Cobbs CS, Harkins L, Samanta M, et al: Human cytomegalovirus infection and expression in human malignant glioma. Cancer Res 62: 3347-3350, 2002.

24. Sadanari H, Yamada R, Ohnishi K, Matsubara K and Tanaka J: SUMO-1 modification of the major immediate-early (IE) 1 and 2 proteins of human cytomegalovirus is regulated by different mechanisms and modulates the intracellular localization of the IE1, but not IE2, protein. Arch Virol 150: 1763-1782, 2005.

25. Zhu H, Shen Y and Shenk T: Human cytomegalovirus IE1 and IE2 proteins block apoptosis. J Virol 69: 7960-7970, 1995.

26. SchwartzR,Helmich B and Spector DH: CREB and CREB-binding proteins play an important role in the IE2 86-kilodalton protein-mediated transactivation of the human cytomegalovirus 2.2-kilobase RNA promoter. J Virol 70: 6955-6966, 1996.

27. Pu P, Kang C, Zhang Z, Liu X and Jiang H: Downregulation of PIK3CB by siRNA suppresses malignant glioma cell growth in vitro and in vivo. Technol Cancer Res Treat 5: 271-280, 2006.
28. Teng MW, Bolovan-Fritts C and Dar RD, et al: An endogenous accelerator for viral gene expression confers a fitness advantage. Cell 151: 1569-1580, 2012.

29. Bai Z, Li L, Wang B, et al: Effect of inducible expressed human cytomegalovirus immediate early 86 protein on cell apoptosis. Biosci Biotechnol Biochem 73: 1268-1273, 2009.

30. Hu M, Wang B, Qian D, et al: Interference with ATF5 function enhances the sensitivity of human pancreatic cancer cells to paclitaxel-induced apoptosis. Anticancer Res 32: 4385-4394, 2012.

31. Chen A, Qian D, Wang B, et al: ATF5 is overexpressed in epithelial ovarian carcinomas and interference with its function increases apoptosis through the downregulation of $\mathrm{Bcl}-2$ in SKOV-3 cells. Int J Gynecol Pathol 31: 532-537, 2012.

32. Augelastro JM, Canoll PD, Kuo J, et al: Selective destruction of glioblastoma cells by interference with the activityor expression of ATF5. Oncogene 25: 907-916, 2006. 